\title{
Selective learning of spatial configuration and object identity in visual search
}

\author{
NOBUTAKA ENDO and YUJI TAKEDA \\ National Institute of Advanced Industrial Science and Technology, Tsukuba, Ibaraki, Japan
}

\begin{abstract}
To conduct an efficient visual search, visual attention must be guided to a target appropriately. Previous studies have suggested that attention can be quickly guided to a target when the spatial configurations of search objects or the object identities have been repeated. This phenomenon is termed contextual cuing. In this study, we investigated the effect of learning spatial configurations, object identities, and a combination of both configurations and identities on visual search. The results indicated that participants could learn the contexts of spatial configurations, but not of object identities, even when both configurations and identities were completely correlated (Experiment 1). On the other hand, when only object identities were repeated, an effect of identity learning could be observed (Experiment 2). Furthermore, an additive effect of configuration learning and identity learning was observed when, in some trials, each context was the relevant cue for predicting the target (Experiment 3 ). Participants could learn only the context that was associated with target location (Experiment 4). These findings indicate that when multiple contexts are redundant, contextual learning occurs selectively, depending on the predictability of the target location.
\end{abstract}

A target defined by salient features, such as color or orientation, can be located more quickly and more consistently, independently of set size. This is referred to as parallel or efficient search (Treisman \& Gelade, 1980; Wolfe, 1994). Natural scenes do not necessarily contain salient bottom-up information. In these cases, the search performance depends on set size; this is referred to as $s e$ rial or inefficient search. Natural scenes often contain too much information to guide attention efficiently (Chun, 2000). Visual context plays an important role in scene perception. A target appearing in a particular scene can be detected more quickly with repeated learning of the scene. In a study of context-learning effects in inefficient search, Chun and Jiang (1998) demonstrated the spatial configuration repetition effect of search items. They compared search efficiency for a repeatedly presented configuration and for a newly generated configuration in an inefficient search task. The results showed that search for the repeatedly presented configuration gradually became faster than search for the newly generated configuration. This facilitatory effect is termed contextual cuing.

\footnotetext{
We thank Jeremy Wolfe, Yuhong Jiang, and an anonymous reviewer for useful comments on earlier versions of this article. This research was supported by Research Fellowships of the Japan Society for the Promotion of Science for Young Scientists to N. Endo. Correspondence should be addressed to N. Endo or Y. Takeda, Visual Cognition Group, Institute for Human Science and Biomedical Engineering, National Institute of Advanced Industrial Science and Technology (AIST), Tsukuba Central 6, 1-1-1 Higashi, Tsukuba City, Ibaraki 305-8566,Japan (e-mail: n-endou@aist.go.jp or yuji-takeda@ aist.go.jp).
}

Note-This article was accepted by the previous editorial team, headed by Neil Macmillan.
Contextual cuing is the guidance of attention on the basis of the implicit memory of an association between a global context (i.e., a spatial configuration) and a target location (see Chun \& Nakayama, 2000). This effect can be observed despite chance performance for recognizing the learned configurations, suggesting that memory for context information is implicit. Moreover, it has been suggested that a complete spatial configuration is unnecessary for spatial cuing to be effective. Spatial cuing depends on learning locally grouped distractors or an attended set of distractors associated with target location (Jiang \& Chun, 2001; Olson \& Chun, 2002).

Visual context also involves other attributes, such as object identities (see, e.g., Biederman, Mezzanotte, \& Rabinowitz, 1982). Chun and Jiang (1999) examined whether a context, based on object identities, would guide visual attention to a target. In their study in which novel shapes were used, the pairing between the locations of a target and distractors was randomized in every trial, but the pairing of identities between the target and the distractor sets was kept constant. They found that significant object-cuing effects, similar to spatial configuration cuing, occurred in this condition. This indicates that visual attention can be guided to a target, as long as the context maintains the association between target and distractor identities.

In these studies, object cuing was independent of the spatial configuration of the search items, because the spatial configuration always changed in the identity repetition condition. However, because spatial cuing and object cuing were investigated in separate studies, it was not clear whether spatial cuing was independent of object identities or whether the two types of contextual learning 
interacted with each other. In the present study, in order to examine the interaction between spatial configuration learning and object identity learning in inefficient search, we manipulated both spatial configurations and object identities, using three repetition conditions: spatial configuration repetition (the configuration repetition condition), object identity repetition (the identity repetition condition), and the combination of both repetition conditions (the combined repetition condition). To assess the contextual-cuing effect, these repetition conditions were compared with a control condition, in which new spatial configurations and new object identity combinations were generated (new condition). In examining object identity learning, in order to control for past experience with the object, 91 novel contours were used as stimuli.

Context learning in a visual search task entails learning an association between target information (e.g., target location or identity) and distractor regularity (e.g., distractor configuration or identities). Classical conditioning is the process of forming representations of an association between a conditioned stimulus (CS) and an unconditioned stimulus (US; Rescorla \& Wagner, 1972). The conditioned response depends on associative strength, and the conditioned response is produced more often when associative strength is greater.

Overshadowing is a phenomenon observed in classical conditioning when multiple CSs are presented. If a strong CS (e.g., a strong light) and a weak CS (e.g., a soft sound) are both conditioned to a conditioned response, the strong CS produces the conditioned response but the weak CS does not (Pavlov, 1927). One explanation of overshadowing is that associative strength is defined by the salience of the CSs (Rescorla \& Wagner, 1972). Furthermore, Mackintosh (1975) has proposed that overshadowing depends not only on the salience of the CS, but also on prior experience and attention.

When a visual context contains different information, such as spatial configurations and object identities (i.e., the combined repetition condition), how will context learning occur? Although classical conditioning and context learning differ in some aspects (e.g., explicit vs. implicit), overshadowing can occur in the combined repetition condition of a contextual-cuing task. To examine what is learned when both spatial configuration and object identities are repeated, in Experiment 1, participants were tested under the combined repetition condition and the new condition in the first four of five epochs of the experimental session; then, in the fifth epoch, the participants were tested under the configuration repetition condition (after the distractor identities had been changed) and the identity repetition condition (after the distractor configurations had been changed). In Experiment 2, we investigated the effect of learning object identities in the identity repetition condition. In Experiment 3, spatialcuing and object-cuing effects were examined in a mixed block. In these three experiments, both the target identity and the target location for each display were fixed in all the repetition conditions. There are four possible correlations between target information and distractor regularity: (1) that between target location and distractor configuration, (2) that between target identity and distractor configuration, (3) that between target location and distractor identities, and (4) that between target identity and distractor identities. In the present study, Correlations 1 and 2 were represented in the configuration repetition condition, and Correlations 3 and 4 were represented in the identity repetition condition. All four correlations were represented in the combined repetition condition. Finally, in Experiment 4, we also examined the occurrence of both cuing effects in the mixed block, in which the distractor configuration was correlated only with the target identity, whereas the distractor identities were correlated only with the target location.

In previous studies (Chun \& Jiang, 1998, 1999), it has been found that spatial configuration learning is based on an association between distractor configuration and target location and that object identity learning is based on an association between the set of distractor identities and target identity. The identities of the target and the distractors were varied in the study of spatial configuration learning, and the locations of the target and the distractors were changed in every trial in the study of object identity learning. However, in the first three experiments in the present study, distractor configuration was correlated with both target location and identity in the combined repetition and the configuration repetition conditions. Distractor identities were also correlated with both target location and identity in the combined repetition and the identity repetition conditions. In these conditions, distractor configuration could cue not only the target location, but also the target identity, and distractor identities could cue not only the target identity, but also the target location. The manipulation of the correlation between target information and distractor regularity is shown in Figure 1.

Although a recognition test for the repeated context would confirm that memory for context information is implicit, no recognition test was performed in the present study. In previous studies of contextual cuing (e.g., Chun \& Jiang, 1998, 1999), the effect of repetition has been examined on the basis of a particular context (spatial configuration or object identities). In the present study, however, three types of contextual information (spatial configuration, object identities, and a combination of these) were repeated in one experimental session, and therefore, it was difficult to present a recognition test with a single criterion. Furthermore, the question of whether contextual information learning is implicit was not of interest in this study. Therefore, we did not conduct a recognition test.

\section{EXPERIMENT 1}

In Experiment 1, we investigated what is learned when both spatial configuration and object identities are re- 


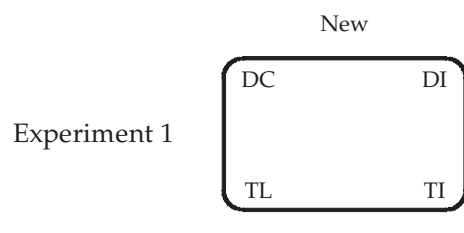

Combined repetition

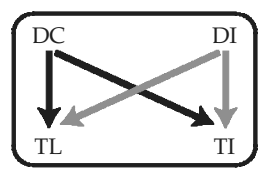

Experiment 2 \begin{tabular}{ll} 
DC & DI \\
& \\
TL & TI \\
\hline
\end{tabular}

Experiment $3 \quad$\begin{tabular}{ll} 
DC & DI \\
& \\
$\mathrm{TL}$ & $\mathrm{TI}$ \\
\hline
\end{tabular}
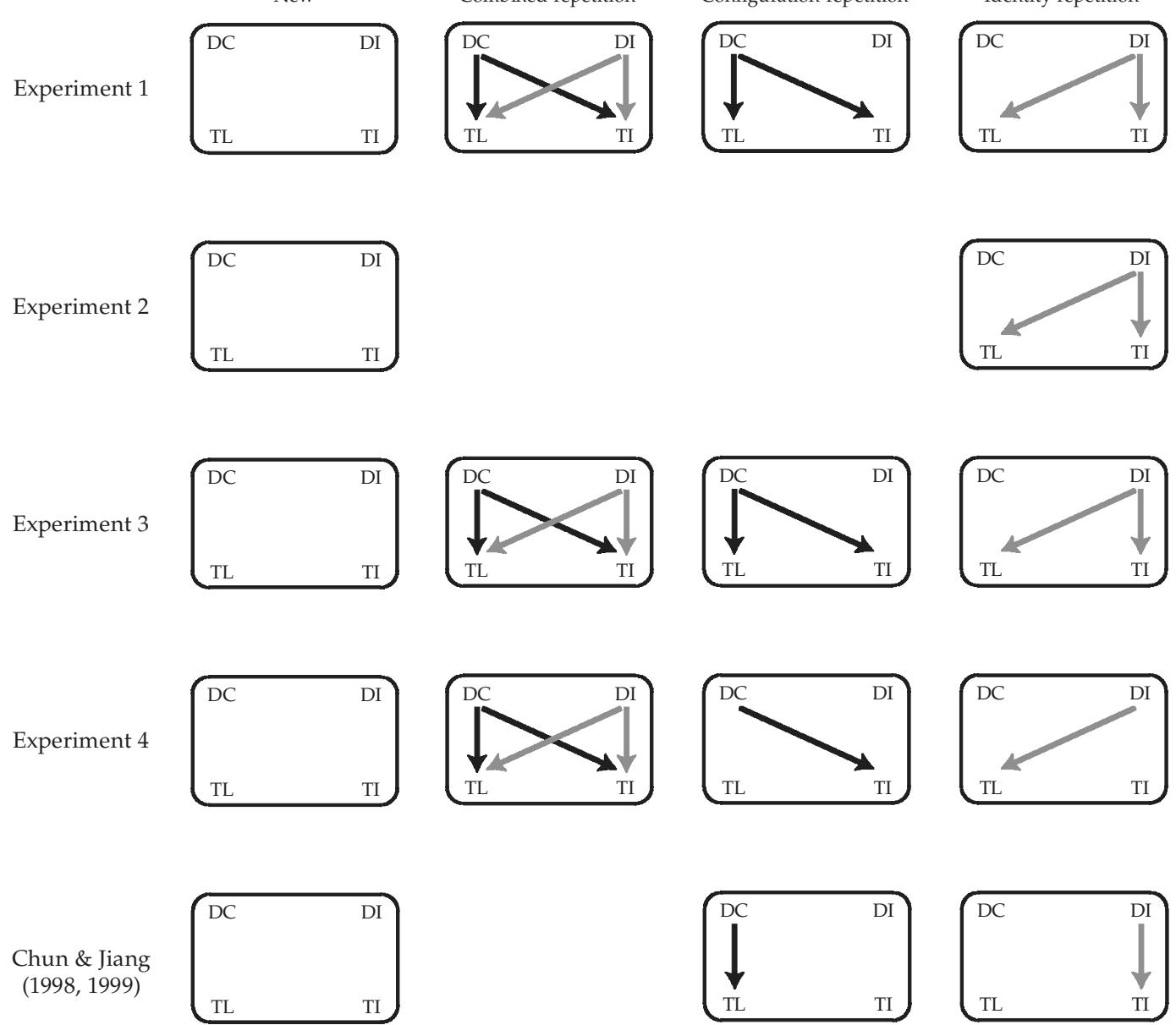

Figure 1. Experimental manipulation of the correlation between target information and distractor regularity. DC, DI, TL, and TI indicate the distractor configuration, the distractor identities, the target location, and the target identity, respectively. The black arrowhead indicates predictions by the distractor configuration, whereas the gray arrowhead indicates predictions by the distractor identities.

peated. The participants were tested under the combined repetition condition in the first four of five epochs of an experimental session, and then either spatial configuration or object identities were varied in the fifth epoch.

\section{Method}

Participants. Fifteen paid volunteers ( 7 males and 8 females; mean age, 21.5 years) participated in Experiment 1. All the participants had self-reported normal or corrected-to-normal vision.

Apparatus and Stimuli. The stimuli were displayed on a 17-in. color monitor (Sony Multiscan 17SF9) with 1,024 × 768 pixel resolution, connected to a computer (Epson Type-HS). The participants responded using a mouse and a numeric keypad, and reaction times (RTs) and responses were recorded on the computer. A program written in MATLAB (The Mathworks, Inc.) controlled the schedule of the experiment. All stimuli were presented on a gray background in a semidark room. The participants observed the stimuli at a $57-\mathrm{cm}$ viewing distance.

In this experiment, we used a total of 91 white novel contours (see Figure 2). All the contours were illustrated by hand (Endo, Saiki, Nakao, \& Saito, 2003). Each contour subtended a visual angle of approximately $2.5^{\circ} \times 2.5^{\circ}$. A target that was defined as a closed contour and nine distractors that were defined as open contours (with a gap that subtended a visual angle of approximately $0.2^{\circ}$ ) were used as search stimuli. The target and the distractors were positioned in 10 randomly selected locations in an invisible $6 \times 6$ matrix that subtended a visual angle of $21^{\circ} \times 21^{\circ}$.

Design. In this experiment, there were three main variables. The first variable was epoch, consisting of five blocks, with a block containing 28 trials. The second variable was the spatial conf iguration of the distractors (repeated and new). The third variable was the set of distractor identities (repeated and new). Thus, there were four types of repetition conditions: the combined repetition, the configuration repetition, the identity repetition, and the new conditions.

A display set consisted of 28 randomly generated configurations and combinations of stimulus identities. In the combined repetition condition, each configuration of the target and the distractors, paired with the identities of the target and the distractors, was repeated throughout the experimental session, once per block (a total of 25 times: 5 epochs $\times 5$ blocks). In the configuration repetition condition, the locations of the target and the distractors were repeated throughout the fifth epoch, while distractor identities were varied from block to block. In the identity repetition condition, the combination of the target and the distractor identities was repeated throughout the fifth epoch, but the distractor locations were ran- 


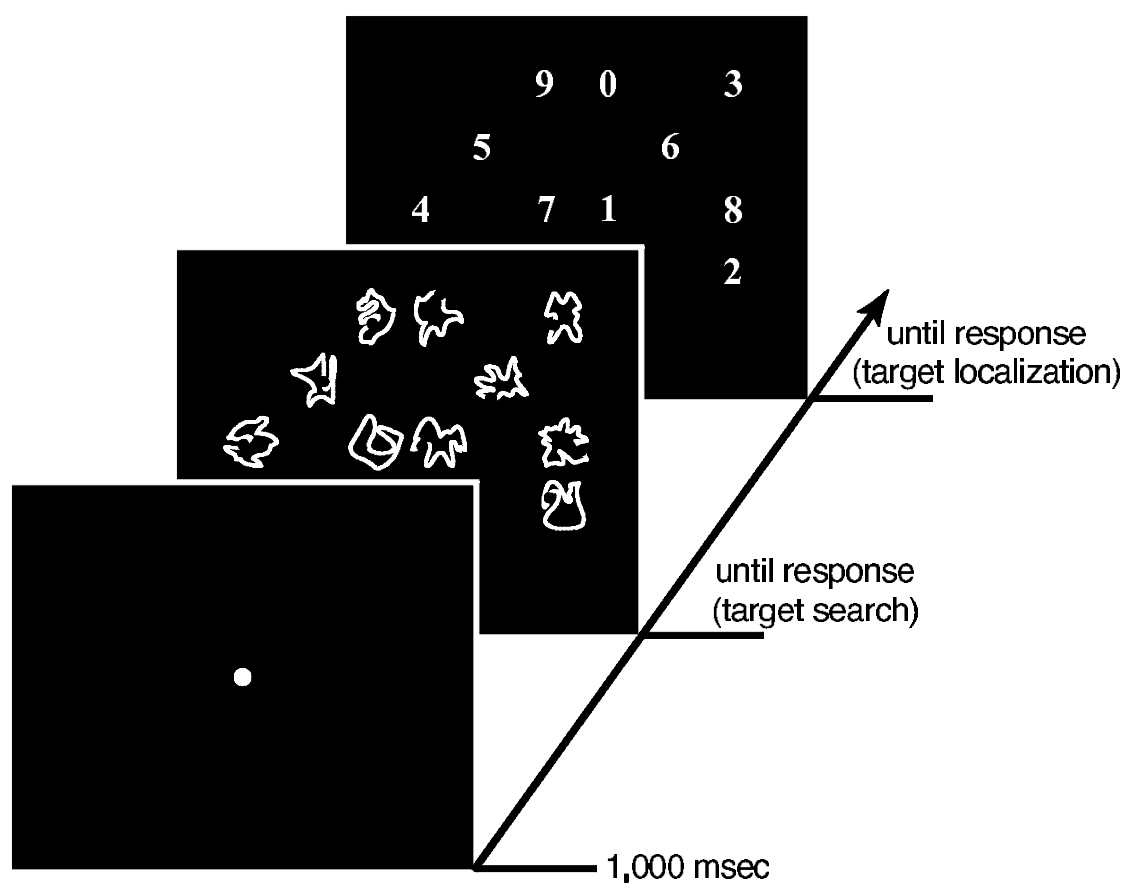

Figure 2. An example of the stimuli used in this study.

domly changed from block to block. Finally, in the new condition, the distractor identities and their locations were randomly chosen in each block to provide a baseline measure. Hence, any difference in performance between the new and the other three conditions could be attributed to the repetition of contextual information based on the spatial configuration of the distractors and/or the set of the distractor identities.

In the study of spatial cuing by Chun and Jiang (1998), the target identity corresponding to a particular repeated configuration was changed from block to block. On the other hand, in the study of object cuing by Chun and Jiang (1999), the target location, as well as the distractor locations, was changed. However, in the first three experiments of this study, the target identity and the target location for each stimulus set were fixed in all the conditions, to prevent a conflict between attentional guidance to the target location and attentional guidance based on the distractor identities.

An experimental session consisted of the training phase (Epochs 1-4) and the test phase (Epoch 5). In the training phase, each participant performed 21 trials of the combined repetition condition and 7 trials of the new condition per block. In the test phase, each participant performed three repetition conditions and the new condition. The 21 trials in the combined repetition condition were equally assigned to the three repetition conditions (i.e., the combined repetition, the configuration repetition, and the identity repetition conditions), each containing 7 trials.

Procedure. Each trial began with the presentation of a white fixation dot at the center of the display. After 1,000 msec from the onset of fixation, a search array was presented. The participants were required to search for a target (i.e., the closed contour) and to respond as quickly as possible by pressing the mouse button. Immediately after a response, the search array was replaced with a target localization array, which consisted of 10 digits (see Figure 2). To confirm the accuracy of the visual search performance, the participants were required to press, as accurately as possible, one of the numeric keys corresponding to the digit positioned in the target location. After this, the display was cleared, and the next trial followed 2,000 msec later. If the target localization was incorrect, a beep sounded as feedback. Before the experimental session, each participant practiced for 28 trials. The distractor configurations and the distractor identities in the practice session were different from those used in the experimental session. The experiment lasted for approximately $1 \mathrm{~h}$.

\section{Results}

All the trials with target localization errors, as well as the trials with target search RTs longer than $6,000 \mathrm{msec}$ (outliers), were excluded from the data analysis (the identical procedure was used in all the subsequent experiments). The data of 2 participants were disqualified from the analysis because more than $10 \%$ of their trials were excluded. The mean correct RTs are shown in Figure 3.

To examine contextual learning in the training phase (i.e., the first four epochs), RTs were subjected to a twoway analysis of variance (ANOVA) with epoch (four epochs) and repetition condition (the combined repetition and the new conditions) as the main terms. The ANOVA indicated that the main effect of epoch was significant $[F(3,36)=32.01, p<.001]$, suggesting that RTs decreased as the training phase progressed. Moreover, there was a significant interaction between epoch and repetition condition $[F(3,36)=3.10, p<.05]$. A Newman-Keuls test indicated that the RTs in Epoch 4 were significantly shorter in the combined repetition condition than in the new condition $(p<.001)$.

We also assessed the effect of changing the distractor configuration and the distractor identities in the test phase. RTs in the test phase were subjected to a two-way 


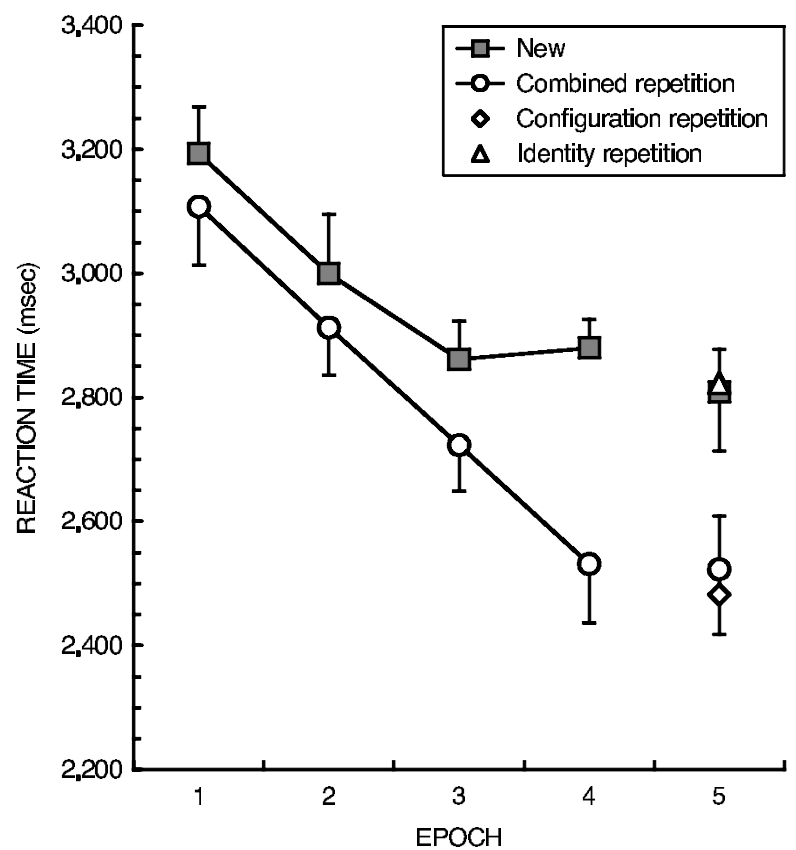

Figure 3. Mean reaction times as a function of epoch for each repetition condition, illustrating the learning function in Experiment 1.

ANOVA with distractor configuration and distractor identities as the main terms. There was a main effect of distractor configuration $[F(1,12)=12.25, p<.005]$, suggesting that RTs were shorter when the distractor configuration was repeated (i.e., the combined and the configuration repetition conditions) than when it was randomly changed (i.e., the identity repetition and the $n e w$ conditions). The other main effect and the interaction were not significant.

Mean error rates as a function of epoch and repetition condition are shown in Table 1. Mean error rates in the training phase were subjected to a two-way ANOVA with epoch and repetition condition as the main terms. There were neither main effects nor an interaction. Thus, the learning effect in RTs could not have been due to a speedaccuracy tradeoff. Mean error rates in the test phase were also subjected to a two-way ANOVA with distractor configuration and distractor identities as the main terms. There were no significant main effects or interactions.

\section{Discussion}

In the first four epochs, RTs in the combined repetition condition were shorter than those in the new condition. Moreover, this advantage increased in the later epochs. These results indicate that contextual information was learned as the experimental session progressed and that this information appropriately guided the participant's attention to a target.

In the fifth epoch, four repetition conditions were examined (i.e., the combined repetition, the configuration repetition, the identity repetition, and the new conditions).
Contextual learning in the training phase completely transferred to the configuration repetition condition. RTs in the configuration repetition condition were not different from those in the combined repetition condition. On the other hand, there was no evidence of transfer to the identity repetition condition. RTs in the identity repetition condition were as long as those in the new condition. These results indicate that the participants could learn the distractor configuration, but not distractor identities, when both were repeated. It is clear that when two redundant contextual cues were presented, one was selectively learned. What determines selective learning? A simple interpretation of our results would suggest that learning a distractor configuration is easier than learning distractor identities. However, according to Rescorla and Wagner (1972), associative strength depends not only on CS intensity, but also on US intensity. In the combined repetition condition, the distractor configuration and distractor identities were correlated with both target location and target identity. Therefore, in context learning, if the US intensity of target location is greater than that of target identity, and if the distractor configuration and distractor identities tend to be associated with target location and target identity, respectively, selective learning might be caused by difference in US intensities. As has been reported in previous studies (Chun \& Jiang, 1998, 1999), in general, participants are likely to learn an association between distractor configuration and target location or an association between distractor identities and target identity. Although the distractor configuration and distractor identities may be completely redundant as cues of target information (i.e., target location and target identity), participants might selectively learn an association between distractor configuration and target loca-

Table 1

Mean Error Rates (\%) as a Function of Epoch and Repetition Condition in This Study

\begin{tabular}{|c|c|c|c|c|c|}
\hline \multirow[b]{2}{*}{ Condition } & \multicolumn{5}{|c|}{ Epoch } \\
\hline & 1 & 2 & 3 & 4 & 5 \\
\hline & \multicolumn{5}{|c|}{ Experiment 1} \\
\hline Combined repetition & 2.20 & 1.39 & 1.47 & 1.90 & 1.10 \\
\hline Identity repetition & & & & & 1.54 \\
\hline Configuration repetition & & & & & 1.98 \\
\hline \multirow[t]{2}{*}{ New } & 2.64 & 2.64 & 1.54 & 2.86 & 1.54 \\
\hline & \multicolumn{5}{|c|}{ Experiment 2} \\
\hline Identity repetition & 2.86 & 2.62 & 2.50 & 1.43 & 1.55 \\
\hline \multirow[t]{2}{*}{ New } & 3.81 & 2.62 & 2.62 & 3.45 & 2.62 \\
\hline & \multicolumn{5}{|c|}{ Experiment 3} \\
\hline Combined repetition & 4.46 & 1.07 & 2.32 & 2.32 & 1.43 \\
\hline Identity repetition & 3.39 & 1.96 & 2.32 & 2.14 & 2.14 \\
\hline Configuration repetition & 2.32 & 2.50 & 1.43 & 1.96 & .71 \\
\hline \multirow[t]{2}{*}{ New } & 3.75 & 1.79 & 2.32 & 1.96 & 1.79 \\
\hline & \multicolumn{5}{|c|}{ Experiment 4} \\
\hline Combined repetition & 2.86 & 1.43 & 2.50 & 1.79 & 3.93 \\
\hline Identity repetition & 3.57 & 2.86 & 2.14 & 1.61 & 1.79 \\
\hline Configuration repetition & 3.57 & 3.21 & 1.43 & 2.50 & 2.32 \\
\hline New & 2.86 & 3.21 & 2.32 & 3.21 & 3.57 \\
\hline
\end{tabular}


tion, because in attentional guidance to a spatial location, target location is more important than target identity. If distractor identities are the only cue for target information, object cuing will be observed. We addressed this issue in Experiment 2.

\section{EXPERIMENT 2}

In Experiment 2, the participants' performances in the identity repetition and the new conditions throughout the experimental session were compared. If the distractor identities could be learned, it was hypothesized that a facilitation effect would be observed in the identity repetition condition.

\section{Method}

Participants. Fifteen paid volunteers ( 9 males and 6 females; mean age, 20.9 years) participated in Experiment 2. All the participants had self-reported normal or corrected-to-normal vision.

Apparatus and Stimuli. The apparatus and stimuli were identical to those in Experiment 1.

Design. The two main variables were epoch (Epochs 1-5) and distractor identities (repeated and new). Thus, the two types of repetition conditions were the identity repetition and the new conditions. Each repetition stimulus set consisted of 14 randomly generated spatial configurations, combined with 10 randomly selected novel contours (one target and nine distractors). There were five epochs, and each epoch consisted of five blocks, with a block containing 28 trials (14 trials for each condition).

Procedure. The procedure was identical to that in Experiment 1.

\section{Results}

The data for 3 participants were disqualified from the analysis because more than $10 \%$ of their trials were excluded. The mean correct RTs are shown in Figure 4.

Correct RTs were subjected to a two-way ANOVA with epoch (Epochs 1-5) and distractor identities as the main terms. The ANOVA indicated that the main effect of epoch was significant $[F(4,44)=25.16, p<.001]$, suggesting that RTs decreased as the experimental session progressed. Moreover, the interaction between epoch and distractor identities was significant $[F(4,44)=2.65$, $p<.05]$. A Newman-Keuls test indicated that there was significant difference between the identity repetition and the new conditions in Epoch $4(p<.05)$.

The mean error rate for each condition as a function of epoch is shown in Table 1. A two-way ANOVA conducted with epoch and distractor identities as the main terms indicated that neither the main effects nor the interaction was significant, suggesting that there was no speedaccuracy tradeoff.

\section{Discussion}

The results of this experiment demonstrated that in the later epochs of the experimental session, the RTs in the identity repetition condition were shorter than those in the new condition, suggesting that object cuing had occurred. These results are consistent with the findings of the previous study by Chun and Jiang (1999).

In the identity repetition condition, Experiments 1 and 2 differed because, in Experiment 2, the participants

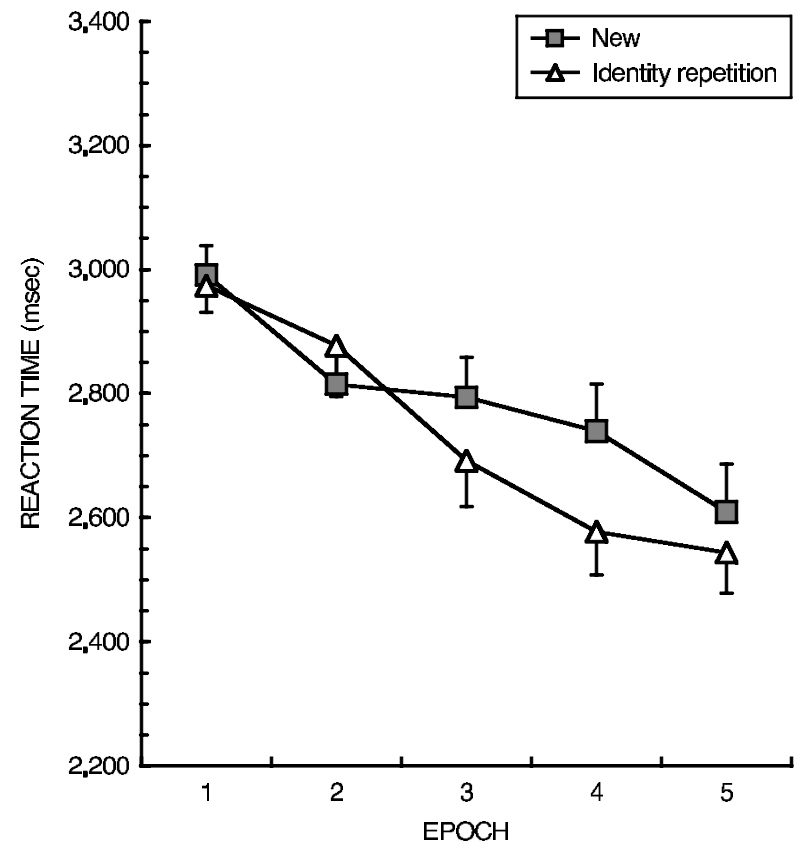

Figure 4. Mean reaction times as a function of epoch for each repetition condition, illustrating the learning function in Experiment 2.

learned the distractor identities with variable configurations, but in Experiment 1, they learned the distractor identities with the identical configuration (i.e., the learning of the distractor identities in the combined repetition condition through the training phase). The object identity learning observed in this experiment is evidence that the absence of object identity learning in Experiment 1 was due to the selection of redundant contextual information. In Experiment 2, object cuing occurred when the distractor identities were the unique cues to the target information. If the four types of repetitions were to appear randomly, would spatial cuing and object cuing occur simultaneously? In this case, the distractor configuration and the distractor identities would not be redundant, because the distractor configurations would be important cues for target information on some trials (i.e., in the configuration repetition condition), and the distractor identities would be important on other trials (i.e., in the identity repetition condition). We addressed this issue in Experiment 3.

\section{EXPERIMENT 3}

In Experiment 3, the participants performed the four types of repetition conditions in a mixed block. Unlike in Experiments 1 and 2, in Experiment 3 each of the contexts based on distractor configuration and distractor identities would be useful in a search for a target. For example, if only the distractor configuration was learned, search performance could be facilitated in the combined repetition and the configuration repetition conditions, 
but not in the identity repetition condition. Thus, learning effects in both the configuration repetition and the identity repetition conditions would be found in this experiment. Furthermore, if each type of contextual information were to be learned independently, spatial cuing and object cuing would be additive in the combined repetition condition.

\section{Method}

Participants. Nineteen paid volunteers (13 males and 6 females; mean age, 20.5 years) participated in Experiment 3. All the participants had self-reported normal or corrected-to-normal vision.

Apparatus and Stimuli. The apparatus and stimuli were identical to those in Experiments 1 and 2.

Design. The three main variables were epoch (Epochs 1-5), distractor configuration (repeated and new), and distractor identities (repeated and new). Each repetition set of stimuli consisted of seven randomly generated spatial configurations (the target location and the distractor locations) paired with 10 randomly selected object identities (one target and nine distractors). There were five epochs, and each epoch consisted of five blocks, with a block containing 28 trials (i.e., 7 trials for each repetition condition).

Procedure. The procedure was identical to that in Experiments 1 and 2 .

\section{Results}

The data for 3 participants were disqualified from the analysis because more than $10 \%$ of their trials were excluded. The mean correct RTs are shown in Figure 5.

Correct RTs were subjected to a three-way ANOVA with epoch, distractor configurations, and distractor identities as the main terms. The ANOVA indicated that the main effect of epoch was significant $[F(4,60)=$ $45.64, p<.001$ ], suggesting that RTs decreased with the progress of the experimental session. The main effect of the distractor identities was also significant $[F(1,15)=$ $6.15, p<.05$ ], suggesting that RTs were shorter when the distractor identities were repeated (i.e., the combined repetition and the identity repetition conditions) than when they were randomly changed. Although the main effect of distractor configuration did not reach significance $[F(1,15)=4.01, p=.064]$, RTs tended to be shorter when the distractor configuration was repeated (i.e., the combined repetition and the configuration repetition conditions) than when it was changed. There was a significant interaction between epoch and distractor identities $[F(4,60)=3.78, p<.01]$. A Newman-Keuls test indicated that RTs were shorter over Epoch 3 when the distractor identities were repeated than when they were changed (all $p \mathrm{~s}<.05)$. The other possible interactions were not significant (all $p s>.10)$.

The mean error rate for each repetition condition by epoch is shown in Table 1. A three-way ANOVA was conducted, with epoch, distractor configuration, and distractor identities as the main terms. The ANOVA indicated that the main effect of epoch was significant $[F(4,60)=6.39, p<.001]$, showing more errors in Epoch 1 than in the subsequent epochs (all $p$ s $<.005$ ). The other main effects and possible interactions were not

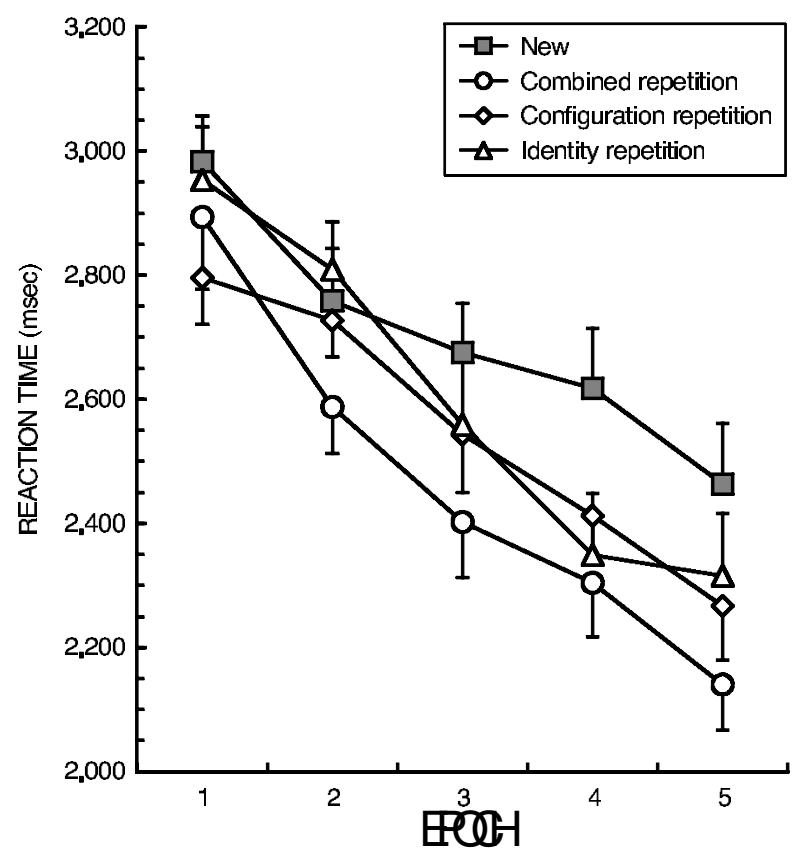

Figure 5. Mean reaction times as a function of epoch for each repetition condition, illustrating the learning function in Experiment 3.

significant, indicating that there was no speed-accuracy tradeoff.

\section{Discussion}

Experiment 3 was conducted to examine whether spatial configuration learning and object identity learning would occur in the configuration repetition condition and the identity repetition condition, respectively, when both conditions appeared in a mixed block. RTs became shorter in the configuration repetition and the identity repetition conditions than in the new condition, although the main effect of the distractor configuration failed to reach significance $(p=.064)$. Furthermore, the interaction between distractor configuration and distractor identities was not significant $(p>.10)$. These results indicated that the spatial cuing and the object cuing were additive. Both spatial cuing and object cuing occurred at the same time when the contexts based on the distractor configuration and the distractor identities were useful cues for searching for a target. On the other hand, when each context of the distractor configuration and the distractor identities was redundant, learning of the redundant context information (i.e., the distractor identities) was blocked (see Experiment 1). In Experiment 3, both the distractor configuration and the distractor identities were useful cues for predicting target information, because the distractor configuration and the distractor identities were unique cues for target information in the configuration repetition condition and the identity repetition condition, respectively. In contrast, in Experiment 1, each 
of two cues was redundant, and only one kind of associative learning was selectively strengthened. This may have occurred through the same mechanism as the overshadowing observed in classical conditioning.

As is shown in the results of Experiments 1-3, we found that contextual learning occurred selectively. However, the determinants of this selectivity were unclear. There are two possibilities for the determinants of selective learning. One is the superiority of the learning of distractor configuration (i.e., the CS intensity). It has been suggested that spatial configuration is more important for the automaticity of attentional control than object identities (Lassaline \& Logan, 1993). Thus, learning of only the distractor configuration might have occurred even if both the distractor configuration and the distractor identities were repeated. Another possibility is that the selection of context learning depends on the information values of a target (i.e., the US intensity). Previous studies have suggested that the association between distractor configuration and target location and the association between distractor identities and target identity can be learned and that these contexts are used in visual search (Chun \& Jiang, 1998, 1999). If one supposes that target location is more useful than target identity in visual search, it is possible that the association between distractor configurations and target locations are stronger than the association between distractor identities and target locations when both contexts are correlated with target locations. We tested these two possibilities in Experiment 4.

\section{EXPERIMENT 4}

In Experiment 4, the participants performed in the four types of repetition conditions in a mixed block. Unlike in Experiment 3, the distractor configuration was correlated only with the target identity (i.e., the configuration repetition condition), and the distractor identities were associated only with the target location (i.e., the identity repetition condition). Thus, in the configuration repetition condition, it was supposed that spatial configuration would predict "what the target is," but not "where it is," and in the identity repetition condition, it was supposed that distractor identities would predict "where the target is," but not "what it is." With these manipulations, it was assumed that distractor configuration would be less informative than distractor identities for attentional guidance to a target location. If selective context learning depends on its informativeness in a search for a target, we would predict an occurrence of learning of distractor identities, which would be informative for attentional guidance to a target location.

\section{Method}

Participants. Twenty paid volunteers (13 males and 7 females; mean age, 21.6 years) participated in Experiment 4. All the participants had self-reported normal or corrected-to-normal vision.

Apparatus and Stimuli. The apparatus and stimuli were identical to those in Experiments 1-3.

Design. The experimental design in Experiment 4 was the same as that in Experiment 3, expect for the following manipulation. In Experiments 1-3, the target location and the target identity in each set were fixed in all the repetition conditions. In Experiment 4, we manipulated the configuration repetition and the identity repetition conditions. The distractor configuration was combined only with the target identity in the configuration repetition condition. In contrast, the distractor identities were combined only with the target location in the identity repetition condition.

Procedure. The procedure was identical to that in Experiments $1-3$, except that the target localization display of digits occupied all $6 \times 6$ cells, but not the 10 previous locations, in order not to repeat the same configuration again with the digit display.

\section{Results}

The data for 4 participants were disqualified from the analysis because more than $10 \%$ of their trials were excluded. The mean correct RTs are shown in Figure 6.

Correct RTs were subjected to a three-way ANOVA with epoch, distractor configuration, and distractor identities as the main terms. The ANOVA indicated that the main effect of epoch was significant $[F(4,60)=26.43$, $p<.001]$, suggesting that RTs decreased with the progress of the experimental session. The main effect of the distractor identities was also significant $[F(1,15)=$ $7.44, p<.05$ ], suggesting that RTs were shorter when the distractor identities were fixed than when they were randomly changed. The other main effect and the possible interactions were not significant. To confirm that distractor identity repetition (the combined repetition and the identity repetition conditions) facilitated target search, we conducted a three-way ANOVA for the first and the last epochs. The ANOVA showed a significant interaction between epoch (Epoch 1 and Epoch 5) and distractor identity repetition $[F(1,15)=5.88, p<.05]$.

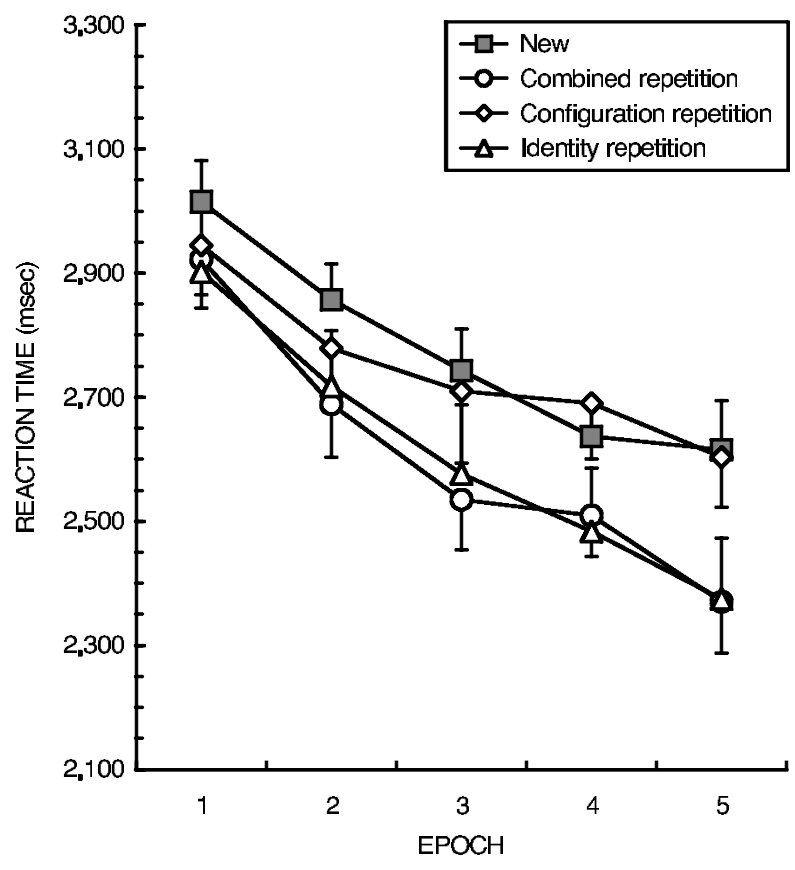

Figure 6. Mean reaction times as a function of epoch for each repetition condition, illustrating the learning function in Experiment 4. 
The mean error rate for each repetition condition by epoch is shown in Table 1. A three-way ANOVA was conducted with epoch, distractor configuration, and distractor identities as the main terms. The ANOVA revealed neither main effects nor possible interactions. Thus, there was no speed-accuracy tradeoff.

\section{Discussion}

The purpose of Experiment 4 was to examine whether selective learning depended on informativeness for target location. The results showed an occurrence of contextual cuing in the combined repetition and the identity repetition conditions. In Experiment 4, target location was fixed in the combined repetition and the identity repetition conditions, thus suggesting that the context associated with the target location was selectively learned. Also, there was no learning effect in the configuration repetition condition, suggesting that the association between the distractor configuration and the target identity could not be learned. Alternatively, the information concerning target identity might not facilitate visual search in this experiment, even if the association between the distractor configuration and the target identity was learned. Therefore, it is possible that the additive effect in Experiment 3 was produced by learning of the association between the distractor configuration and the target location and the association between the distractor identities and the target location. These results suggested that context learning would be selected on the basis of the predictability of the target location.

\section{GENERAL DISCUSSION}

The purpose of this study was to examine the association between spatial configuration learning and object identity learning in inefficient search. The results of Experiment 1 showed that only spatial configuration was learned when both spatial configuration and object identities were predictive of target information (i.e., both target location and identity). However, it was demonstrated in Experiment 2, that object identity learning could occur when only object identities were predictive of target information. Furthermore, in Experiment 3, object identity learning occurred even when the configuration repetition condition was presented. The results of Experiments 1 and 3 showed that the occurrence of context learning of distractor configuration and distractor identities is selective. As was shown in the results of Experiment 3, an additive learning effect of two contexts could be found when both spatial configuration and object identities were useful for search. Furthermore, it is thought that the occurrence of selective context learning depended on the informativeness of the context in cuing target location. Both the distractor configuration and the distractor identities could be used as a cue to target location, whereas the distractor configuration was not available for use as a cue to target identity (see Experiment 4). Chun and Jiang (1999) have suggested that object cuing occurs when an association be- tween distractor identities and target identity is learned. However, in this study, it has been suggested that an association between distractor identities and target location can be learned. The inconsistency of these findings may result from the difference between the search tasks - the vertical symmetry search task in Chun and Jiang's (1999) study and the closed contour search task in the present study. Further investigations are needed to clarify this issue.

According to the results of Experiment 4, it is possible that the occurrence of context learning depends on an association between distractor configuration and target location and an association between distractor identities and target location. In Experiment 1, both of these associations appeared repeatedly; therefore, the association between the distractor identities and the target location might have been redundant, because it is likely that the association between the distractor configuration and the target location was stronger than the association between the distractor identities and the target location. Thus, learning of the more informative context might have blocked the learning of the less informative context. In contrast, in Experiment 2, there was nothing to block the learning of the less informative context, because only the object identities appeared repeatedly. Furthermore, in Experiment 3, when each of the spatial configurations and the object identities was informative in some trials, blocking was reduced. Hence, spatial configuration learning and object identity learning occurred at the same time, and the additive effect could appear in the combined repetition condition. Finally, in Experiment 4, context learning occurred only in the identity repetition condition, but not in the configuration repetition condition, because only the distractor identities were predictive of the target location. Target location is the most critical information in a visual search task, and it is likely that context information that predicts a target location will be learned. The results of this study show that "what is predicted" is more important for contextual cuing than is "what is repeated." The absence of identity learning in Experiment 1 may be explained by the similar phenomenon of overshadowing in classical conditioning studies. However, obviously, further work is needed to clarify the relationship between selective learning in contextualcuing tasks and overshadowing in classical conditioning.

In summary, we believe that people can use two types of contextual information to guide attention to a target in inefficient search — namely, spatial configuration and object identities. However, even when both contexts are predictive of the target, people will be likely to select the more useful context of the two, rather than learning both contexts. On the other hand, if both contexts are useful for search, the additive effect of spatial configuration learning and object identity learning can be observed. In general, our findings support the idea that not all of the available regularity and invariance are used by the cognitive system, because the implicit learning mechanism must operate with a limited capacity. 


\section{REFERENCES}

Biederman, I., Mezzanotte, R. J., \& Rabinowitz, J. C. (1982). Scene perception: Detecting and judging objects undergoing relational violations. Cognitive Psychology, 14, 143-177.

Chun, M. M. (2000). Contextual cueing of visual attention. Trends in Cognitive Sciences, 4, 170-177.

CHUn, M. M., \& JIANG, Y. (1998). Contextual cueing: Implicit learning and memory of visual context guides spatial attention. Cognitive Psychology, 36, 28-71.

Chun, M. M., \& JiAng, Y. (1999). Top-down attentional guidance based on implicit learning of visual covariation. Psychological Science, 10, 360-365.

Chun, M. M., \& NAKAYAMA, K. (2000). On the functional role of implicit visual memory for adaptive deployment of attention across scenes. Visual Cognition, 7, 65-81.

Endo, N., SAIKI, J., NAKaO, Y., \& SaITo, H. (2003). Perceptual judgments of novel contour shapes and hierarchical descriptions of geometrical properties. Japanese Journal of Psychology, 74, 346-353.

JiANG, Y., \& CHUN, M. M. (2001). Selective attention modulates implicit learning. Quarterly Journal of Experimental Psychology, 54A, 1105-1124.

Lassaline, M. E., \& Logan, G. D. (1993). Memory-based automatic- ity in the discrimination of visual numerosity. Journal of Experimental Psychology: Learning, Memory, \& Cognition, 19, 561-581.

MACKINTOSH, N. J. (1975). A theory of attention: Variations in the associability of stimulus with reinforcement. Psychological Review, 82, 276-298.

Olson, I. R., \& Chun, M. M. (2002). Perceptual constraints on implicit learning of spatial context. Visual Cognition, 9, 273-302.

PAVLov, I. P. (1927). Conditioned reflexes: An investigation of the physiological activity of the cerebral cortex (G. V. Anrep, Trans.). London: Oxford University Press.

ResCorla, R. A., \& WAGNER, A. R. (1972). A theory of Pavlovian conditioning: Variations in the effectiveness of reinforcement and nonreinforcement. In A. H. Black \& W. F. Prokasy (Eds.), Classical conditioning II: Current research and theory (pp. 64-99). New York: Appleton.

Treisman, A., \& Gelade, G. (1980). A feature-integration theory of attention. Cognitive Psychology, 12, 97-136.

Wolfe, J. M. (1994). Guided Search 2.0: A revised model of guided search. Psychonomic Bulletin \& Review, 1, 202-238.

(Manuscript received November 28, 2002; revision accepted for publication September 3, 2003.) 